\title{
Research on Kinetic Waves and Applications
}

\author{
Luciano Rota ${ }^{1}$ \\ ${ }^{1}$ Sportmark 104; 1355 KH Almere (NL), the Netherlands \\ Correspondence: Luciano Rota, Sportmark 104; 1355 KH Almere (NL), the Netherlands. Tel: 316-4143-0644. \\ E-mail: lmrrota@live.nl \\ Received: March 20, 2017 \\ Accepted: April 26, 2017 \\ Online Published: May 12, 2017 \\ doi:10.5539/apr.v9n3p8 \\ URL: https://doi.org/10.5539/apr.v9n3p8
}

\begin{abstract}
The duality waves/particle and the dynamic of propagation of electromagnetic emanations suggest the existence of a natural kind of waves, which differently from de classic ones, are originating by kinetic thrust and propagating, also though vacuum, by inertial force. The model taken into consideration, to which has been given the name of "kinetic waves" is, like the classic one, a concretely existing natural phenomenon which can also be visually perceived if produced on molecular scale. Results suggest, by giving consistent mathematical proof, that kinetic waves offer many more points of similarity, in dynamic and behavior, than the classic ones, which were taken, since the discovery of electromagnetic waves, as basic model.

Applying the obtained results relatively to this model, to the astrophysical red-shift, taking as example the quasar $3 \mathrm{C}-273$ and the recently found, most far galaxy GN-z11, we could find a mathematical sustainable and logic answer about still unsolved problems with regard to the origin and the dynamic of the universe.

In the appendix, a suggested and accurately described experiment on base of Radar Astronomy to possibly confirm the validity of this model.
\end{abstract}

Keywords: light propagation, classic waves, kinetic waves, particles, Doppler-shift, redshift

\section{Introduction}

\subsection{About Waves}

Around the end of the17th century Isaac Newton argued that light consists of small particles or corpuscles. By his "Corpuscular Theory" he sustained that those particles were literally shouted from the source in the form of beams. Basically a ballistic theory, as we should call it today.

Christian Huygens (1629-1695), the Dutch mathematician, physicist and astronomer, in the same time, formulated the "Huygens principle", nowadays better known as Huygens-Fresnel principle, and generally argued that light consists of waves. He connected the dynamics of light-waves to that of sound-waves.

In 1865 the Scottish physicist James Maxwell, arrived by experiment to the formulation of the Theory of the Magnetic Fields, concluding that light propagates in form of waves through space. This discovery sustained Huygens's theory, so that Newton's Corpuscular Theory, was finally disregarded.

The common concept of "waves" is connected to vibrations of matter through the matter itself. So we could without doubt state that, in the classical concept of waves, these must necessarily make use of a basic material - liquid, solid, gassy, etheric (as it was speculated before 1887) or finally in de form of magnetic fields (as theorized today) - to be able to propagate; since the very concept of "waves" contains a dynamic of vibration of matter through matter itself. It is understood that the conclusions of Maxwell naturally referred to the classic concept of waves as above described (according to the Huygens-Fresnel hypothesis), that also include the principle of Doppler Effect as a result of variation of frequencies caused by the movement of the light source through space, with respect to an observer.

It is a fact that all theories of Modern Physics and quantum mechanics that have followed till nowadays, are originally - based and further developed, on the wavy dynamics connected to the sound-waves model of propagation. That means: constancy of the speed of propagation and variation of the wavelength as an increase or decrease of the originally emitted frequencies, when the source moves through space. 
In other words, the whole modern physics is sustained by the pillars of the constancy of the speed of light and the fact that this remains unchanged regardless the movements of the source with respect to the observers.

The path of scientific research that has followed during the last century to the present day, has certainly not been free of contradictions and doubts on the validity/consistency of mathematical-physics theories that have gradually met in the course of more than a century. Many problems have been solved by means of quantum theories and by the "Standard Model" with regard to the Particles Physics, but certainly not all of them. Especially those with regard to Astrophysics and Cosmology, where some important interrogatives are still without an answer.

Of course, as long as the non-constancy of light-speed won't be finally proven, things remain unchanged and the theoretical research must go on, although, we may consider that Science, till date, has never taken into consideration that electromagnetic waves may not be connected to the classic ones. As previously announced, there is in nature a different kind of waves with a strong similarity in structure, behavior and dynamic of propagation with the electromagnetic ones, that concretely could explain all unsolved problems, especially with regard to Astrophysics and Cosmology, in a logic and sustainable way.

This research aims to give of it an analytical description and mathematical proof.

\section{Doppler Shift on Base of Sound Waves}

Regarding the classic Doppler, we consider two different aspects: a) when an emitting source is moving to or from a stationary observer or: b) when an observer is moving to or from a stationary emitting source. Just for clarity, we shall call a) Doppler 1 and b) Doppler 2

\section{Doppler 1}

Treating of classical waves (sound waves), we have to consider that an objective variation of the wavelength can be registered when a source is moving through a matter. Let us take a look at the following figure (1):

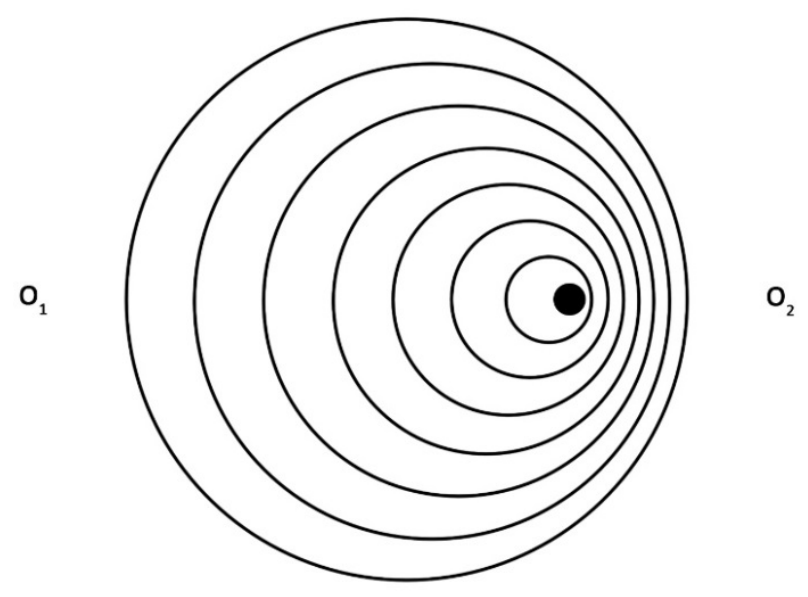

velocity of source relative to observers is to the right

Figure 1. Doppler-shift 1: source moves to/from observers

As we can see, the movements of the source through the matter produce a real, objective increasing or decreasing of the wavelength, so that the observer, at a constant speed of propagation, receives an increased or decreased frequency.

In case of increase or decrease of wavelength, the observer will receive a frequency:

$$
f_{o}=f_{e}\left(\frac{v_{u}}{v_{u} \pm v}\right)
$$

( $f_{o}=$ observed frequency; $f_{e}=$ original emitted; $v_{u}=$ velocity of waves through matter; $v=$ velocity of source with regard to observers.) 


\section{Doppler 2}

We examine the case when the observer is moving to a stationary source of waves (Figure 2):

When the emitting source is stationary, we see that there is no difference between wavelength emitted and wavelength observed:

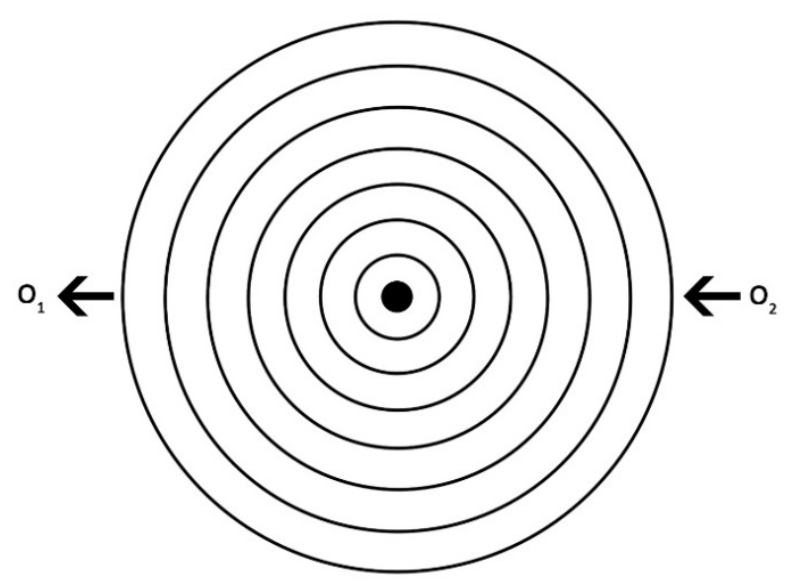

velocity of source relative to observers is 0

Figure 2. Doppler-shift 2:observers move to/from source

By observing this last figure, it is evident that we are dealing with a completely different phenomenon than that described in Figure 1 What we can see in Figure 2 is that when a source is stationary there is no variation of wavelength, but a difference in frequency the observer subjectively records due to the relative motion between the latter and the source. In this case the frequency observed has been calculated by:

$$
f_{o}=f_{e}\left(1-\frac{v}{v_{u}}\right) \quad \text { when the observer is regressing from source. }
$$

And:

$$
f_{o}=f_{e}\left(1+\frac{v}{v_{u}}\right) \quad \text { when is approaching. }
$$

Or:

$$
f_{o}=f_{e}\left(\frac{\left.v_{u} \pm v\right)}{v_{u}}\right.
$$

( $f_{e}=$ frequency original emitted; $f_{o}=$ frequency obs. $; v_{u}=$ speed of waves through matter; $v=$ speed of the observers with regard to source)

a) Speaking of sound waves, in this case the source emits a constant wavelength (Figure2) which is proportional to the speed- or frequency - of vibrations of the source (a wire, for example.) $=f_{\mathrm{v}}$ and the constant speed of propagation $\left(v_{u}\right)$. The moving observer at velocity $v$ receives a frequency, which is the number of wave-tops, transmitted through the atmosphere, the observer receives in a measure of time at constant speed of propagation:

$$
\lambda_{e}=\frac{1}{f_{v}} v_{u} \rightarrow f_{o}=\frac{1}{\lambda} v_{u}
$$

in absence of relative motion between source and observer, at constant speed of propagation: 


$$
f_{v}=f_{o} \text { and } \lambda_{e}=\lambda_{o}
$$

To make it more clear, let us see the following example:

Let's say that a wire is vibrating at a frequency $f_{v}=500 / \mathrm{s}$; Constant speed of propagation $v_{u}=300 \mathrm{~m} / \mathrm{s} ; v=0$ :

$$
\lambda_{e}=\frac{1}{500} 300=0,6 \rightarrow f_{o}=\frac{1}{0,6} 300=500
$$

b) By relative motion between source and observer the wavelength remains constant (Figure2) but the frequency undergoes a variation relatively to the value of the speed $\mathrm{v}$ :

$$
f_{o}=\frac{1}{\lambda_{e}}\left(v_{u} \pm v\right) \text { and: } \lambda_{e}=\lambda_{o}=\lambda
$$

Now, the observer moves towards the source at a speed $v=20 \mathrm{~m} / \mathrm{s}$ :

$$
f_{o}=\frac{1}{0,6}(300+20)=533,33
$$

The difference in observed frequency calculated by Doppler 1 (eq. 1) and Doppler 2 (eq. 2, 3 or 4) is very small when dealing with a low speed $v$, but it grows quadratic the more the difference between $v$ and $v_{u}$, decreases:

By Doppler 1, using Equation 1, when the source is moving towards a stationary observer at the same speed $v=$ $20_{m / s:}$

$$
f_{o}=f_{e}\left(\frac{v_{u}}{v_{u}-v}\right)=f_{o}=500\left(\frac{300}{300-20}\right)=535,71
$$

As already mentioned the difference between the results in observed frequency by Doppler 1 and Doppler 2 increases the more the speed $v$ approaches that of $v_{u}$. Taking in the same example a velocity $v=40 \mathrm{~m} / \mathrm{s}$, by Doppler 2: $f_{o}=566,66$; and by Doppler 1: $f_{o}=576,92$. Increasing the speed $v$ to $80 \mathrm{~m} / \mathrm{s}$, by D2: $f_{o}=633,33$; by D1: $f_{o}=681,81$.

To resume: Doppler 1 modifies the frequency on base of the variation of the value of wavelength. Doppler 2 modifies the frequency on base of the value of relative motion $(v)$ between source and observer.

\section{Kinetic Waves and Duality Waves/Particles}

The original Corpuscular Theory of Newton is, as told, basically a ballistic theory. Newton argued that light was made up of particle beams projected from the source. He, in his time, had not the notion of the fact, found a century later by Maxwell, that the light propagated in the form of waves. For over a century it is known that the composition of the light beams consists in a duality of waves and particles.

In classical physics the principle of "waves" is connected to the concept of rippling of the material by the material itself; sound, movements of water which propagate across its surface or the vibrations that run along a wire.

What the three kinds of waves described above have in common is the fact that they need a material substance through which to propagate, be it in a gassy, liquid or solid state. The speed of these waves is, therefore, calculated in relation to the material substance in which they occur.

Electromagnetic waves, in several aspects, are not similar to the above-mentioned waves. There are some important differences, like:

1) It appears that they do not need a field of any kind in which to propagate:

When we speak about "Doppler-shift" we implicitly speak of rippling of material substances through the matter self. Any theorizing referring to the Doppler 1 must be connected to classical waves in the sense above explained.

2) Electromagnetic emanation consists of both waves and particles. 
The structure of magnetic waves on the field of research can never be considered as a synthetic phenomenological context. Research can just be made on particles or, separately, on waves, treating (on field of research) the two parts of the same energy emanation as two different phenomena.

Differently, classical waves can be contained in a single context: there is a matter and rippling of the matter self.

3) Regarding the Doppler Effect, there is not any difference in observed frequency, when the source is moving from the observer or vice versa.

\subsection{Method.}

We integrate those data in a single context, in order to obtain an image of what the structure and the nature of magnetic waves concretely could be on ground of Newton's Theory and the model of Doppler 2:

The most relevant data which we can use is the knowledge of the fact that the particles making up matter contain a vibratory motion. It is also well-known that the speed of these vibrations is directly proportional to the degree of heat of the matter in a relation that in rough synthesis we may define thus: the hotter the matter the faster its particles vibrate, the higher the frequencies it emanates.

Now let us imagine that, due to kinetic thrust, these particles are literally fired from electrons into space in the form of continuous jets, at the original constant speed - in relation to the source - of approx. 300 thousand $\mathrm{Km} / \mathrm{s}$. With regard to the fact that the electrons have a vibratory movement, the result that we would obtain is that of rippling fluxes, or better of particle waves, whose original wavelength would vary in relation to the degree of heating of the source emitting them. (as solved by eq. 5)

This figure shows what a vibrating electron would look like:

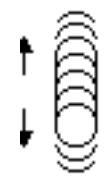

Figure 3. add a title here

It is already well known that each electron sends photons. Let us imagine that those small particles together have been shot in a continuing flux from vibrating electrons. Then we see something like this:

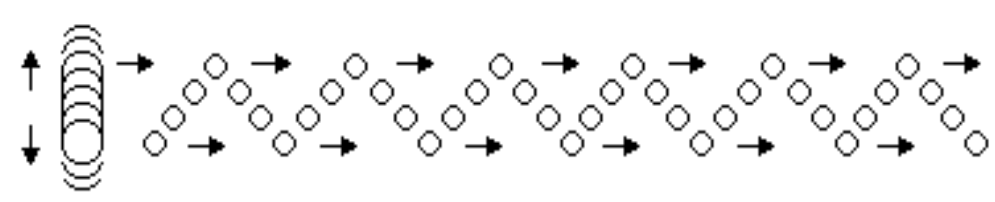

Figure 4. add a title here

$$
\lambda_{e}=\frac{1}{f_{v}} c
$$

Although each small particle follows a straight line, all parts together give the flux a waving motion. This would give a concrete explanation of the fact that research on the field of electromagnetic emission have to consider waves and particles separately from each other: if we take a look at figure 4 we clearly see that every single particle follows a straight line, so that when researching particles, it is impossible to get an idea of a wavy structure. If not, when researching waves, we must synthetize the particle emission in a global wavy flux.

Heated matter is never heated uniformly: usually the nucleus is the part most heated. The temperature gradually decreases towards the external parts of the matter. Making a relation between thermic degree and speed of the 
particles' vibration, we would logically find that the highest frequencies would be emanated from the hottest layers while the lowest from the coldest.

That means, the hotter the matter, the faster the electrons vibrate, the shorter the wavelength of the waving flux, so that the distance between two wave tops becomes smaller:

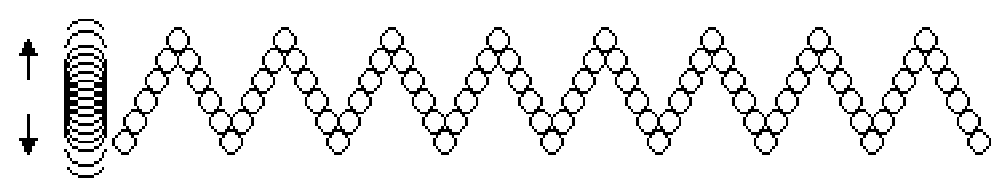

Figure 5. add a title here

$$
\lambda^{\prime}{ }_{e}=\frac{1}{f_{v}{ }^{\prime}} c \quad\left(f_{v}{ }^{\prime}>f_{v}\right)
$$

This model offers us the following conclusions:

1) Waves do not need any material substance in which to propagate. Since they originate from the source that produces them, they can propagate even through vacuum and proceed by inertial force. In the absence of gravity and agents of attrition, we could suppose that the speed originally imparted and so the wavelength remains unvaried (constant) to the infinite.

2) From this point of view we can see how the duality of emission regarding waves and particles can be totally and concretely explained: looking at this structure we can easily conclude that we are dealing with waves and with particles emanation as well. In fact, the particles are making_up a wavy flux. This would be a concrete way to connect waves and particles emission in a synthetic phenomenological context conform to Newton's Corpuscular Theory and Maxwell's field equations.

3) Regarding Doppler 2, in this hypothesis the behavior of the waves in relation to the frequency variations ascribable to the relative motion is perfectly coherent with the premise: the variation of frequency recorded in relation to the source's movements with respect to the observer, or vice versa, are not a consequence of Doppler 1 effect: in the sense that they do not represent a variation of wavelength, but a variation of the registered frequency, caused by the relative increase or decrease in relative speed of the flux between source and observer.

Frequency is depending on movements of the source with regard to an observer. Which means that every variation of the emission speed - due to a performing relative speed - will be perceived by an observer as a variation of frequency.

Dealing with electromagnetic waves, using the formulas relative to Doppler 2 we can observe:

a) The wavelength emitted is related to the frequency of vibrations of the electrons and the constant speed of propagation:

( $f_{v}=$ frequency of vibrations $)$

$$
\lambda=\frac{1}{f_{v}} c
$$

b) The stationary observer receives a frequency:

$$
f_{o}=\frac{1}{\lambda} c
$$

c) The moving source relatively to the observer or the moving observer with regards to the source at a relative velocity $v$ receives a frequency:

$$
f_{o}=\frac{1}{\lambda}(c \pm v)
$$


Comparing electromagnetic waves to kinetic waves, as above explained, we expect that the variation in frequency we register when the source moves to a stationary observer, or when the latter moves to a stationary source, are exactly the same, as we can show by these results:

a) The observer is moving away from the source: speed of vibration $=\mathrm{f} / \mathbf{s}$. Speed of the flux $=v_{u}$. velocity of the observer $=v$. The distance between two wave-tops is: $d=\frac{1}{f}$ of $v_{u}$

$$
\begin{aligned}
& d=\frac{v_{u}}{f} \\
& O=a_{o}+v t \\
& W_{1}=b_{o}+v_{u} t \\
& W_{2}=b_{o}-d+v_{u} t \\
& W_{2(\Delta t)}=O_{\Delta t} \Rightarrow b_{o}-d+v_{u} \Delta t=a_{o}+v_{\Delta t} \\
& W_{1(o)}=O_{(o)} \Rightarrow b_{o}=a_{o} \\
& W_{2(\Delta t)}=O_{\Delta t} \Rightarrow b_{o}-d+v_{u} \Delta t=a_{o}+v_{\Delta t} \\
& -d=v_{\Delta t}-v_{u(\Delta t)}=\left(v-v_{u}\right) \Delta t \Rightarrow \Delta t=\frac{-d}{v-v_{u}} \\
& \Delta t=\frac{v_{u}}{\left(v_{u}-v\right) f} \\
& f_{o}=\frac{1}{\Delta t}=\frac{\left(v_{u}-v\right) f}{v_{u}}
\end{aligned}
$$

b) The source is moving away from the observer:

Speed of the flux + distance

$$
\begin{aligned}
& d^{\prime}=\frac{v_{u}-v}{f}+\frac{v}{f} \\
& d^{\prime}=\frac{v_{u}-d v+v}{f}=\frac{v_{u}}{f} \\
& W_{1}^{\prime}=a_{o}+\left(v_{u}-v\right) t \\
& W_{2}^{\prime}=a_{o}-d^{\prime}+\left(v_{u}-v\right) t \\
& O^{\prime}=0 \\
& \left(\begin{array}{l}
W_{1(o)}^{\prime}=O^{\prime}{ }_{(o)} \Rightarrow a_{o}=0 \\
W_{o(\Delta t)}^{\prime}=O^{\prime}{ }_{(\Delta t)} \Rightarrow a_{o}-d^{\prime}+\left(v_{u}-v\right) \Delta t=0
\end{array}\right) \rightarrow \\
& \rightarrow d^{\prime}+\left(v_{u}-v\right) \Delta t=0 \\
& \Delta t\left(v_{u}-v\right)=d^{\prime} \\
& \Delta t=\frac{d^{\prime}}{\left(v_{u}-v\right)} \\
& f_{o}^{\prime}=\frac{1}{\Delta t}=\frac{\left(v_{u}-v\right)}{d^{\prime}} \Rightarrow f_{o}{ }^{\prime}=\frac{\left(v_{u}-v\right)}{v_{u}} f
\end{aligned}
$$

If the source approaches the observer the sign will be $(+)$

In both cases (source moves to observer or vice versa, approaching or moving away) will be as solved in (4).

c) In absence of relative motion between source and observer the wavelength is constant and at constant speed of propagation the frequency observed will be the same of the original emitted vibration frequency: 


$$
f_{o}=\frac{1}{\lambda} c=f_{v}
$$

To resume:

1) Sound waves are originating by the vibrations of a source (a wire for example) which emits a wavelength that is directly proportional to the speed of the vibration. Sound waves propagate trough a matter - at a constant speed - which can change the structure of the wavelength by moving through the atmosphere. The wavelength remains constant when the source is stationary, but an observer can receive a lower or higher number of wave-tops in the same measure of time, as consequence of the relative motion between source and observer. The frequency observed, in the two cases, is different. A difference which is very small, when dealing with low relative speed, but increasing on square scale the more the difference between speed of propagation and that of relative motion become smaller.

2) Kinetic waves (by original model) are beams of particles originating by kinetic thrust, from a vibrating source (think of the wavy effect of a flow of water produced by a vibrating garden hose), which emit a wavelength that is directly proportional to the speed of vibration. Kinetic waves propagate at constant speed through matter (atmosphere) and trough vacuum as well, by inertial thrust. The wavelength remains constant even when the source is moving through space, but the observed frequency changes by effect of the relative motion between source and observer (eq. 8). Kinetic waves make no difference in observed frequency whether the source moves to the observer or vice versa.

\subsection{Experimental Results}

The following mentioned experimental results:

Michelson-Morley

Fizeau convection coefficient

Kennedy-Thorndike

Moving sources and Mirrors

\section{Aberration}

since they are performed on ground of the movements of sources through space, in absence of relative motion between source and observer, did not register any different observed frequency than that original emitted. (as solved by eq. 7) On this point we can also take into account the calculations made by Walther Ritz in his ballistic Emission Theory, published in 1908 (Ritz, 1908; Ritz, 1908; Tolman, 1910).

By kinetic waves, as we have seen, the constancy of the speed of emitted wavy light-beams and the constancy of the wavelength, give as result that the frequency remains unchanged in observation (as solved by eq. 9).

Experimental results like De Sitter Spectroscopic Binaries which disagree with ballistic theories, are starting from the ground of Doppler 1 which is based on the constancy of propagation speed of electromagnetic waves, and the variations of wavelength.

De Sitter Spectroscopic Binaries is the most mentioned experimental result in disagree with Ritz emission theory. Just to remind:

"According to simple emission theory, light thrown off by an object should move at a speed of $c$ with respect to the emitting object. If there are no complicating dragging effects, the light would then be expected to move at this same speed until it eventually reached an observer. For an object moving directly towards (or away from) the observer at $v$ meters per second, this light would still be expected to be travelling at $(c+v)$ or $(c-v)$ at the time it reached us.

In 1913, Willem de Sitter argued that if this was true, a star in a double-star system would usually have an orbit that caused it to have alternating approach and recession velocities, and light emitted from different parts of the orbital path would then travel towards us at different speeds. De Sitter made a study of double stars and found no cases where the stars' computed orbits appeared. Since the total flight-time difference between "fast" and "slow" light-signals would be expected to scale linearly with distance in simple emission theory, and the study would (statistically) have included stars with a reasonable spread of distances and orbital speeds and orientations, De Sitter concluded that the effect should have been seen if the model was correct, and its absence meant that the emission theory was almost certainly wrong." 


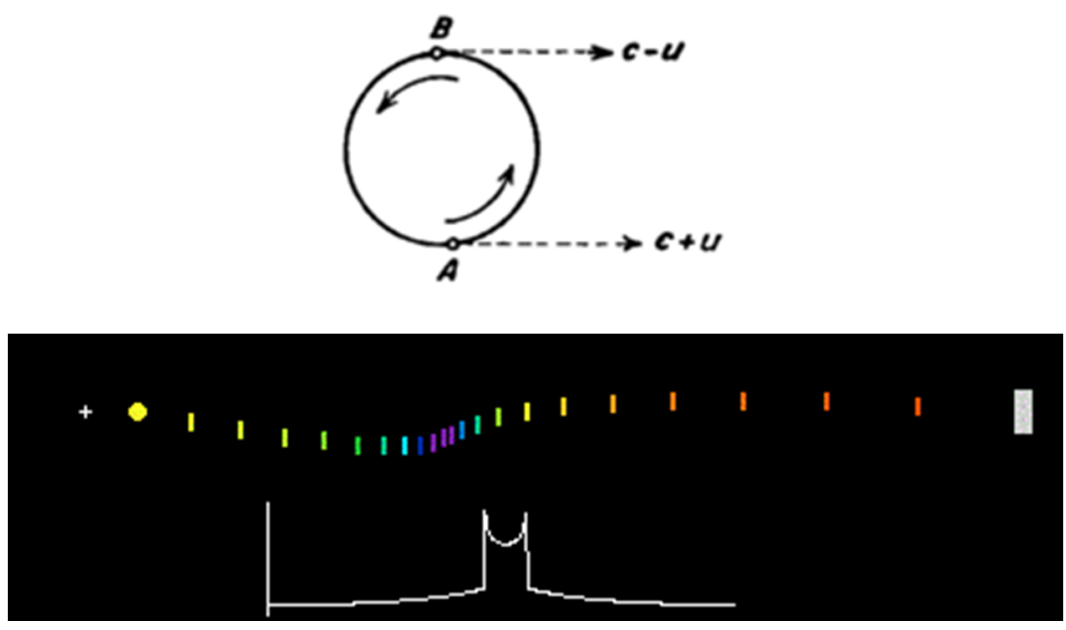

Figure 6

Comparing electromagnetic waves to classic waves the arguments deducted by De Sitter's astronomical observation, would be certainly correct. It won't be correct whether we connect them to the model of kinetic waves and Doppler 2.

As we take a look at Figure 6, the expected extra increasing of frequency due to the relative speed of approaching of the source is calculated on decreasing of the wavelength (Doppler 1), adding the increasing of relative speed $v$ (Doppler 2) so that the conclusion deducted by this experiment is calculated by a measuring made on ground of variations of wavelength due to approaching speed plus that of increased relative speed:

$$
f_{o}=f_{e}\left(\frac{c}{c-v}\right)+\frac{v}{c} f_{e}
$$

Which was not in line to the expected results by Ritz's model, because, on base of Doppler 2 and kinetic waves, the variation in frequency must be calculated just on ground of the variation of the observed speed of emission: ( $c$ $+v)$ or $(c-v)$. So that we expect that when the source is moving to/from us the observed frequency will be given by:

When the source is approaching:

$$
f_{o}=\frac{1}{\lambda}(c+v)
$$

when the source is regressing:

$$
f_{o}^{\prime}=\frac{1}{\lambda}(c-v)
$$

From this angle we also expect that these two results will be constantly alternate each other in approach and recession velocities. We don't expect any ulterior increasing or decreasing of speed of the fluxes, just because the variations in speed is already taken into account as only consequence of the differences in observed frequency between approaching of de source and regressing of it.

\section{Redshift as Progressive Decreasing of Light-Speed on Travelled Distance}

We make use of Doppler 2 model to recalculate the redshift, starting from the premise that the emitting sources finding them self at very great distances from us are stationary and the frequency we observe registers a decreasing with regard to the original emitted, due to a decreased original emitted light-speed, proportionally to the travelled distance. Whether light-beams travel by inertial force through great distances, the original kinetic thrust could be 
decreased by effect of gravity fields they have to cross or scattered atomic waste present in space that crossed at very high speed (that of light) can offer a substantial material consistency.

\subsection{Results}

Let us consider the following examples:

\section{Example 1:}

We take as first the quasar known as 3C-273:

(for these calculations has been used a round light-speed of $c=300.000 \mathrm{Km} / \mathrm{s}$ )

\section{Calculation of the redshift of 3C-273 on base of Doppler 1}

Doppler 1 fixes the constant factor in the value of speed of propagation and the variable one in the value of wavelength.

The hydrogen Ballmer-alpha line in stationary stand registers a wavelength of $656_{\text {n.m. }}$. The observed wavelength of this body in $760_{\text {n.m. }}$. Calculating redshift and recession velocity on base of Doppler 1:

$$
\begin{gathered}
\lambda_{e}=656_{n, m} ; \lambda_{o}=760_{n, m} ; z=\frac{\lambda_{o}-\lambda_{e}}{\lambda_{e}}=0,1585 \rightarrow v=(c z)=47550 \mathrm{~km} / \mathrm{s} \quad \text { (recession speed on base of wavelength) } \\
f_{c}=457_{T H z} ; f_{o}=394_{T h s} ; z=\frac{f_{e}-f_{o}}{f_{o}}=0,1585 \rightarrow v=(c z)=47550 \mathrm{~km} / \mathrm{s} \quad \text { (on base of frequency) }
\end{gathered}
$$

The calculation of the redshift this way, would be possible till $z<1$. When $z>1$, the regression speed will be greater than that of light. If we take the quasar 5C 02.56 discovered in 1970 (Illingworth, 1999), it shows a redshift of: $z=2,399$, corresponding to a regression speed of: $v=719.700 \mathrm{~km} / \mathrm{s}$; GB1428 +4217 (Space Daily, 2012) $=\mathrm{z}=$ 4,72 and recession speed $=1.416 .000 \mathrm{~km} / \mathrm{s}$; GRB090423 $(\mathrm{NASA}, 2009)=\mathrm{z}=8,2$ and recession speed $=$ $2.460 .000 \mathrm{~km} / \mathrm{s}$. Going on to the most recent time, the galaxy GN-z11 (Drake, 2016), which shows a redshift calculated in $\mathrm{z}=11,09$, on base of Doppler 1, it would pretend to move away from us at a speed of more than 3,5 million $\mathrm{km} / \mathrm{s}$.

The Law of Hubble, which is based on the calculations of Doppler 1, is grounded on the astronomical observations and relative spectrum analysis made since 1929. At the time of Hubble's publication, the most distant observable body was the galaxy NGC-7619 (Trimble, 1996) which registered a redshift of 0,012 and a regression speed of about $3.700 \mathrm{~km} / \mathrm{s}$ : a surprising result, at that time, but still contained in the limits allowed by Relativity.

\subsection{Calculation of the Redshift of 3C-273 on Base of Doppler 2.}

Doppler 2, differently from Doppler 1, considers the observed result of frequency, based on constancy of the wavelength and progressive decreasing of light-speed. In other terms and according to what explained about the original Doppler 2 model: the source produces a wavelength and the observer receives a frequency, which is directly proportional to the value of decreased light-speed. This way, is to understand that the redshift would not be consequence of a progressive regression of light-sources on distance, relatively to the observer, but to a decreasing of the observed light-speed which results in a decreased frequency.

According to that, we have to suppose that $656_{\text {n.m. }}$. remains constant in emission as in observation. In stationary stand and at constant speed of emission, an observer will receive a frequency:

$$
f=\frac{1}{\lambda} c=457_{T H z} \text { and } \quad \lambda_{e}=\lambda_{o}=\lambda=656_{n \cdot m}
$$

In case of decreasing of original emission-speed to $(c-v)$ :

$$
f=\frac{1}{\lambda}(c-v)
$$

Now we don't know yet the value of $v$, so we have to fix it on base of which we think to be an observed wavelength. As told, the Hydrogen Ballmer-alpha line relative to this body is on $760_{\text {n.m. }}$. which correspond to a decreased observed frequency of $394_{\mathrm{THz}}$. It means that when on spectrum appears the line on $760_{\text {n.m. }}$, in fact we are receiving 
a frequency of $394_{\mathrm{THz}}$. Since the calculations are programmed on base of Doppler 1, the result on frequency is automatically translated into a value of increased wavelength, although the frequency effectively observed is the value we really register and in this model, we need to reach the value of decreasing in velocity $(v)$. So we can state: the original emitted wavelength (656) is the same observed and the corresponding emitted frequency (457) is decreased by effect of decreased original emission speed to $c-v$ :

$$
z=\frac{f_{o}-f_{e}}{f_{e}}=\frac{394-457}{457}=-0,1378 \rightarrow v=c z=-41.340 \mathrm{~km} / \mathrm{s}
$$

In this case the negative sign of the shift doesn't mean blue-shift, but the value that must be detracted from the original emitted speed. This way:

$$
c_{o b s}=(c-v)=300.000-41340=258.660 \mathrm{~km} / \mathrm{s}
$$

Now we obtained the value of $v$, we can complete the eq. 14 with the missing value:

$$
f_{o}=\frac{1}{\lambda}(c-v)=\frac{1}{656}(258660)=394_{T H z}
$$

As already explained, this value of decreased observed frequency $\left(394_{\mathrm{THz}}\right)$ which by Doppler 1 will be automatically interpreted as an increased wavelength of $760_{\text {n.m. }}$. from this angle it expresses a value of decreased light-speed.

\section{Example 2:}

4.3 Calculation of the Redshift of the Galaxy GN-z11, on Base of Doppler 2.

The redshift of this body is calculated in $\mathrm{z}=11,09$ :

$$
\lambda=656 \rightarrow f=457
$$

The Hydrogen-Ballmer-alpha-line signs a wavelength on $8.462_{\text {n.m. }}$ which corresponds to a frequency:

$$
\begin{gathered}
f_{o}=\frac{c}{\lambda_{o}}=\frac{300.000}{8462}=35,5_{\text {THz }} \\
z=\frac{f_{o}-f_{e}}{f_{e}}=\frac{35,5-457}{457}=-0,9223 ; v=c z=(300.000)(-0,9223)=-276.695 \\
f_{o}=\frac{1}{\lambda}(c-v)=\frac{1}{656}(300.000-276695)=35,5_{\text {THz }}
\end{gathered}
$$

Whit regard to the galaxy GN-z11, is to suppose that objects finding them self on distances $>30$ Gly would be impossible to be optical perceived, because the observed frequency will be decreased below the optical frequency limit.

The results obtained by the calculations on base of Doppler 2 model, taking as example 3C-273 and GN-z11, would give theoretical proof that none of the cosmic objects we can optically perceive is regressing.

\section{Conclusion}

The model of kinetic waves (KW), we have described and analyzed in the present theoretical research, in addition to a correct mathematical analysis based on classic mechanics, offers many more points of similarity with the behavior of electromagnetic waves and correct results than the waves model offered by the classic model.

1) $\mathrm{KW}$ are expected to behave in full agreement with the experimental results which confirm there is no difference in variation of frequency whether a source is moving to a stationary observer or vice versa.

2) KW could give a concrete explanation about the duality particles/waves, in the way that wavy fluxes consist in particles beams 
3) KW do not need any material substance or magnetic field through which propagate, since they are sent by kinetic thrust and proceeding by inertia through vacuum.

4) $\mathrm{KW}$, as basically connected with a ballistic emission theory, agree with all experimental results performed on this field. KW offers a correct, sustainable and logical answer about the causes of the redshift, next to the classic physical laws we know.

The relation travelled distance/redshift, in the described and analyzed way would give us the image of a globally static universe and as consequence, the indication that light coming from the most distant celestial bodies undergoes a slowdown which is directly proportional to the distance from us: the bigger the distance that light have to travel to reach us, the stronger the slowdown, the sharper the redshift.

The "Big-Bang Theory" is grounded on the statement made by Hubble in 1929, about the principle of universal expansion. When this ground would be missing, there will not be any other reason to sustain the universe originated by an enormous explosion of a concentrated cosmic matter. However, it will exonerate us to find or imagine explanations with regard to where and how each cosmic object finds the necessary energy to move from all others in a constant, progressive acceleration to all direction and find a way to meet again at a common point . Besides, through this way would be useless to try giving an age to our Universe or fix an origin of it, which probably never took place.

\section{Suggested experiment:}

Assuming that variations between emitted and observed frequencies are consequence of variations of emitted lightspeed and not of those of wavelength it would be possible to perform the following experiment, based on the speed of revolution of earth around the sun, with regard to an external body. In this example has been taken Jupiter as model. Our planet, related to the orbit of Jupiter presents two phases: one with sign plus $(+)$ when the earth is approaching Jupiter, for example in March, and one with sign minus (-), when the earth is regressing from it, in September.

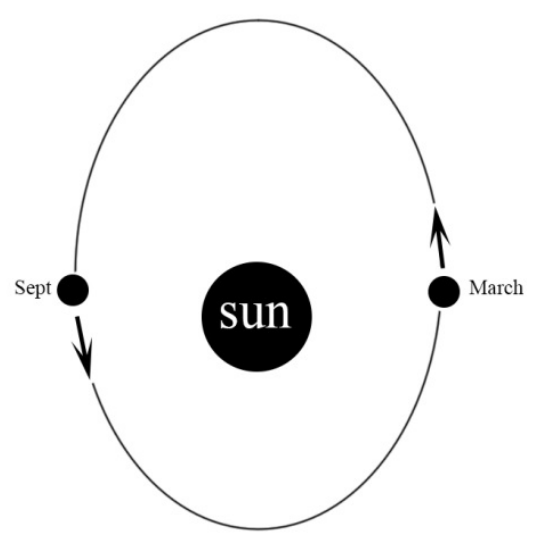

Figure 7

\section{Results}

$C=299.792,458$

Velocity of earth's revolution $(v)=30 \mathrm{~km} / \mathrm{s}$

Average distance Earth/Jupiter $(s)=588.000 .000 \mathrm{Km}$.

Using radar astronomy by sending a beam of microwaves in March in direction of Jupiter, the distance we have to calculate it to reach Jupiter and reflecting back will be:

$$
S=1.173 .000 .000 \mathrm{~km} \text {. }
$$

To recover this distance at light-speed $(C)$ the signal would take: 


$$
\frac{s}{c}=\frac{1.173 .000 .000}{299.792 .458}=3922^{\prime \prime}, 7137
$$

If we add the average rotation's speed of Earth around the sun in March, we obtain:

$$
\frac{s}{(c+v)}=\frac{1.173 .000 .000}{299.822,458}=3922^{\prime \prime}, 3212
$$

Corresponding to a frequency variation calculated by:

$$
f_{o}=\frac{1}{\lambda}(c+v)
$$

Repeating the same experiment on September:

$$
\frac{s}{(c-v)}=\frac{1.173 .000 .000}{299.762,458}=3923 ”, 1063
$$

And a frequency variation:

$$
f_{o}=\frac{1}{\lambda}(c-v)
$$

The difference in terms of time between March and September would be $8 / 10^{\text {th }}$ of a second: when this result would support the expectation, will confirm that the differences of frequency produced by the movements of a source are ascribable to the relative motion between source and observer. This kind of experiments, meant to a direct measuring of the speed of light on base on distance and time taken to cover it, in the age of Modern Physics, has never been performed.

\section{References}

Tolman, R. C. (1910). The second postulate of relativity. Physical Review (Series I), 31(1), 26.

De Sitter double star experiment. (2016). In Wikipedia, the free encyclopedia. Retrieved from https://en.wikipedia.org/wiki/De_Sitter_double_star_experiment

Drake, N. (2016). Astronomers Spot Most Distant Galaxy-At Least For Now. National Geographic.

Illingworth, G. (1999). Galaxies at high redshift. Astrophysics and Space Science, 269, 165-181.

NASA. (2009). New Gamma-Ray Burst Smashes Cosmic Distance Record.

Ritz, W. (1908). Recherches critiques sur l'électrodynamique générale. Annales de Chimie et de Physique, 13, $145-275$.

Ritz, W. (1908). Recherches Critiques sur les Théories Électrodynamiques de Cl. Maxwell and de H.-A. Lorentz. Archives des Sciences physique et naturelles, 26, 209-36.

Space Daily. (2012). Record-Setting X-ray Jet Discovered.

Trimble, V. (1996). H0: The incredible shrinking constant, 1925-1975. Publications of the Astronomical Society of the Pacific, 108(730), 1073-1082.

\section{Copyrights}

Copyright for this article is retained by the author(s), with first publication rights granted to the journal.

This is an open-access article distributed under the terms and conditions of the Creative Commons Attribution license (http://creativecommons.org/licenses/by/4.0/). 